\title{
ERRATUM
}

\section{A genome-wide association study of antidepressant response in Koreans}

W Myung, J Kim, S-W Lim, S Shim, H-H Won, Seonwoo Kim, Sangha Kim, M-S Lee, HS Chang, J-W Kim, BJ Carroll and DK Kim

Translational Psychiatry (2015) 5, e672; doi:10.1038/tp.2015.173; published online 3 November 2015

Correction to: Translational Psychiatry (2015) 5, e633; 10.1038/tp. 2015.127; published online 8 September 2015

In the Acknowledgments, DK Kim was presented incorrectly. In the Author Contributions section, J-WK was involved in study supervision. Additionally, the corresponding authors were presented in the wrong order. The correct order appears below.

Dr DK Kim, Department of Psychiatry, Samsung Medical Center, Sungkyunkwan University School of Medicine, 81 Irwon-ro, Gangnam-gu, Seoul 135-710, Korea.
E-mail: paulkim@skku.edu; Dr J-W Kim, Department of Laboratory Medicine and Genetics, Samsung Medical Center, Sungkyunkwan University School of Medicine, 81 Irwon-ro, Gangnam-gu, Seoul 135-710, Korea.

E-mail: kimjw@skku.edu

The publisher regrets these errors. 